\title{
The Effects of Age, Viewing Distance and Font Type on the Legibility of Chinese Characters
}

\author{
Linghua Ran ${ }^{1, *}$, Xin Zhang ${ }^{1}$, Xiaoyuan Ren $^{2}$, and Huimin $\mathrm{Hu}^{1}$ \\ ${ }^{1}$ China National Institute of Standardization, Zhichun Road, 4, \\ Haidian District, Beijing 100088, China \\ ${ }^{2}$ Shandong Normal University, Wenhua East Road, Jinan, Shan \\ Dong Province, 250014, China \\ ranlh@enis.gov.cn
}

\begin{abstract}
This study evaluated the effects of age (30s and 55s), viewing distance $(50 \mathrm{~cm}, 80 \mathrm{~cm}$ ), font type (Lanting in simple, Lanting in complex, Yahei in simple, Yahei in complex) on the legibility of Chinese characters by using the two legibility measures (minimum character size for $100 \%$ correctness and minimum character size for the least comfort). Twenty subjects in each age group read the 12 word groups (two characters in each word group, character size varied from $4.5 \mathrm{pt}$ to $18 \mathrm{pt}$ ) presented on a paper. Subjects subjectively rated the reading legibility of the word groups on a 3-point scale (1- indistinct, 2- distinct but need cost a little attention, 3- very distinct). According to the ANOVA procedure, age and viewing distance significantly affected the two dependent variables $(p<0.05)$. The younger group could see character sizes smaller than the old group could and the viewing distance of $50 \mathrm{~cm}$ showed character about $1 \mathrm{pt}$ smaller than those at a 80 $\mathrm{cm}$ viewing distance. There are no significant differences in font types. From a comparison of the results for correctness and comfort, people generally preferred larger character sizes to those that they could read. The findings of this study may provide basic data and guidelines for setting of character size and font type to improve the legibility of characters written in Chinese.
\end{abstract}

Keywords: age, viewing distance, font type, Chinese character.

\section{Introduction}

The legibility and readability of characters are important to our daily activities and tasks. If the sizes of the characters are too small or poorly designed, people may not obtain the correct information. The main factors which affect legibility are font size, type of font, thickness of font, space between fonts, contrast of colors, distance of sight, illumination and so on. Many researchers have studied the effects of font size, font type, viewing distance, age and contrast to the legibility of characters.

In a study of font sizes, Wang Jian et al. found the complexity of Chinese characters influence the Chinese character threshold [1].Zhou Aibao et al. explored processing

\footnotetext{
* Corresponding author.
} 
speed influenced by the font, size, and characteristics of Chinese character [2]. The results show that among seven kinds of fonts, processing speed of Sungti, Zhengkai and Heiti is faster than that of Xingkai, Lishu, Weibei, and Caiyun. Japanese researchers have provided a standard for the various font sizes of Japanese characters in visual displays to make them legible to older and visually impaired people [3]. The US also has a standard on character sizes for the labeling of medical supplies [4].

Font type is also a significant factor for the legibility of words in hard copy. Wang You et al. measured the character recognition threshold or acuity of Chinese characters [5]. The results show that four types of Chinese character fonts (Songti, Heiti, Kaiti and Fangsong ) have different acuities: Heiti being the smallest, Kaiti larger, and Songti and Fangsong the largest. The Gothic fonts were smaller than the Ming fonts in Korean users' visual performance which tested two age groups (20s and 60s) for different font types of Korean letters and numbers at $50 \mathrm{~cm}$ and $200 \mathrm{~cm}$ viewing distances respectively [6]. An experiment was conducted to compare with recognition accuracy of four fonts, of Chinese characters under three various back illumination [7]. The results show the Song font and Black font were better than imitative Song font and Long Song font, and Long Song font was worse than imitative Song.

Age and viewing distances are main factors to affect the legibility of characters, but most studies of the effect factors for Chinese character are focus on the font, size, stroke and structure. Therefore, this study investigated the effects of age and viewing distance on the legibility (correctness) and subjective preference (comfort) of Chinese characters associated with different age groups. The objectives of this study were to: (1) provide guidelines for the minimum Chinese character size for $100 \%$ correctness; (2) suggest guidelines for comfortable Chinese character size on the basis of the age of the person.

\section{Methods}

\section{$2.1 \quad$ Subjects}

In all, 40 participants, comprising 20 young people (in their 30 s) and 20 elderly people (in their 55s) were recruited, respectively, to investigate legibility in relation to age. The mean age and mean visual acuity of the young participants were $32.85( \pm 6.05)$ years and $0.84( \pm 0.15)$ respectively, whereas the mean age and mean visual acuity of the elderly groups were $56.90( \pm 4.15)$ years and $0.36( \pm 0.15)$, respectively. All subjects were tested and selected by a Landholt acuity test with a $30 \mathrm{~cm}$ distance measure. All participants were native Chinese speakers. None was color blind or had eye defects.

\subsection{Procedure}

Before the experiments, the personal data of the participants was asked. Then all participants were provided with a brief description of the goals and procedures of this study. The illuminance level was measured and recorded and to confirm it was in an appropriate range at the surface on which the test charts will be placed. And each participant's visual acuity was tested.

After the visual acuity test, each participant was asked to try to read characters presented on papers and determine the reading legibility of the word groups. And the 
participants rated the legibility by using a 3-point scale (1- indistinct, 2- distinct but need cost a little attention, 3- very distinct). The numbers of correct/wrong answers as well as the subjective reading legibility ratings of each character group were recorded. If a participant provided all wrong answers for the two characters twice consecutively, the experimental trial was stopped. After a trail, participant needed to report the least comfort character group depending on character size.

The measurement of the dependent variables were as follows: (1) 'minimum character size for $100 \%$ correctness', which was defined as the smallest font size among character sizes at which the participant correctly read all two characters in each group on papers; (2) 'minimum character size for the least comfort', which was defined as the smallest font size among character sizes at which the participant reported that the characters caused comfort while reading; The ' $100 \%$ ' was employed to determine the minimum character size associated with age groups in this study.

\subsection{Apparatus}

A room was constructed specifically for this study and the environment was comfortable for participants. Two light sources which can control the intensity of illumination were located on the ceiling of the room. To test the legibility of the characters for paper, the surface illumination level was $50 \mathrm{~lx}$ in this study.

Each participant was instructed to read 12 sets of characters (two-syllable Chinese characters) shown on papers at viewing distances of $50 \mathrm{~cm}$ and $80 \mathrm{~cm}$. Unmeaningful words were employed for two-syllable Chinese characters in this study(Fig. 1 shows an example). Altogether, 12 character sizes, ranging in size from $4.5 \mathrm{pt}$ to $18 \mathrm{pt}$, were tested in this study. The spatial complexity of the stimuli was also described by stroke frequency [8]. In this study, characters that contain 10 or more than 10 strokes were described as "complex" characters; and characters that contain 1 to 9 strokes as "simple" characters. Lanting is similar to Yahei, however, there are still difference between them.

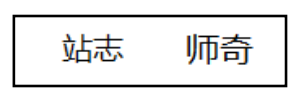

Yahei in complex

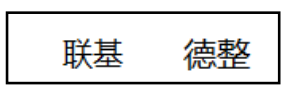

Lanting in complex

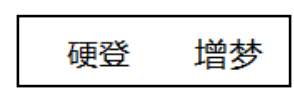

Yahei in simple

列是 和点

Lanting in simple

Fig. 1. An example of two-syllable Chinese characters

\subsection{Experimental Design}

The independent variables were age, viewing distance and font type. Levels for the independent variables are described in Table 1. Each participant performed 8 trials and all treatment combinations were performed in a random order. 
This study investigated the effects of these independent variables on the following dependent variables. For measurement of the dependent variables: (1) 'minimum character size for $100 \%$ correctness'; (2) 'minimum character size for the least comfort'. To evaluate the effects of various independent variables [age $(30 \mathrm{~s}, 55 \mathrm{~s})$, viewing distance $(50 \mathrm{~cm}, 80 \mathrm{~cm}$ ), font type (Lanting in simple, Lanting in complex, Yahei in simple, Yahei in complex], on the two dependent variables, an ANOVA was employed.

Table 1. Description of the independent variables

\begin{tabular}{ll}
\hline Independent variables & Levels \\
\hline Age & $30 \mathrm{~s}, 55 \mathrm{~s}$ \\
Viewing distance & $50 \mathrm{~cm}, 80 \mathrm{~cm}$ \\
Font type & $\begin{array}{l}\text { Lanting in simple, Lanting in complex, Yahei in simple, } \\
\text { Yahei in complex }\end{array}$ \\
\hline
\end{tabular}

\section{$3 \quad$ Results}

\subsection{Analysis of Minimum Character Size for $100 \%$ Correctness}

The effects of age and viewing distance on the minimum character size for $100 \%$ correctness were found to be statistically significant (all p-values $<0.05$ ). There is no significant difference in font types.

In Figure 2, the 'minimum character size for $100 \%$ correctness' for people in their $30 \mathrm{~s}$ was smaller $(6.3 \mathrm{pt})$ compared to the people in their $55 \mathrm{~s}(8.8 \mathrm{pt})$. Put more simply, young participants were able to correctly read character sizes smaller than those elderly participants with the same level of accuracy. The effect of viewing distance on the 'minimum character size for $100 \%$ correctness' was also evidently significant. Font sizes of $6.8 \mathrm{pt}$ and $8.2 \mathrm{pt}$ were reported as the 'minimum character sizes for $100 \%$ correctness' at a $50 \mathrm{~cm}$ viewing distance and at a $80 \mathrm{~cm}$ viewing distance, respectively. Though not significantly, the results of the 'minimum character size for $100 \%$ correctness' associated with the font type indicated that Yahei in simple (7.3 pt) and Yahei in complex $(8.0 \mathrm{pt})$ allowed for smaller character sizes than Lantinghei in simple (7.6 pt) and Lantinghei in complex (8.4 pt) respectively.

\subsection{Analysis of Subjective Minimum Character Size for the Least Comfort}

Statistical analysis showed that age and viewing distance were significant factors for the 'minimum character size for the least comfort' (all p-values < 50.05). 'Minimum character size for the least comfort' means that the smallest font size among character sizes was such that a participant rated the character(s) as causing comfort while reading. There is no significant difference in font types.

Figure 3, showing the results of the main effects of 'minimum character size for the least comfort', indicates that the younger group described characters up to $8.8 \mathrm{pt}$ in size as causing no discomfort to read, whereas the elderly group required a character size of at least $11.0 \mathrm{pt}$ to read characters without discomfort. As expected, the effect of viewing 
distance was significant. The 'minimum character sizes for the least comfort' were 9.3 pt and $11.5 \mathrm{pt}$ for $50 \mathrm{~cm}$ and $80 \mathrm{~cm}$ viewing distances, respectively. Though not significantly, the 'minimum character sizes for the least comfort' associated with the font type indicated that Yahei in simple (9.5 pt) and Yahei in complex (9.9 pt) allowed for smaller character sizes than Lantinghei in simple (10.3 pt) and Lantinghei in complex (10.5 pt) respectively.

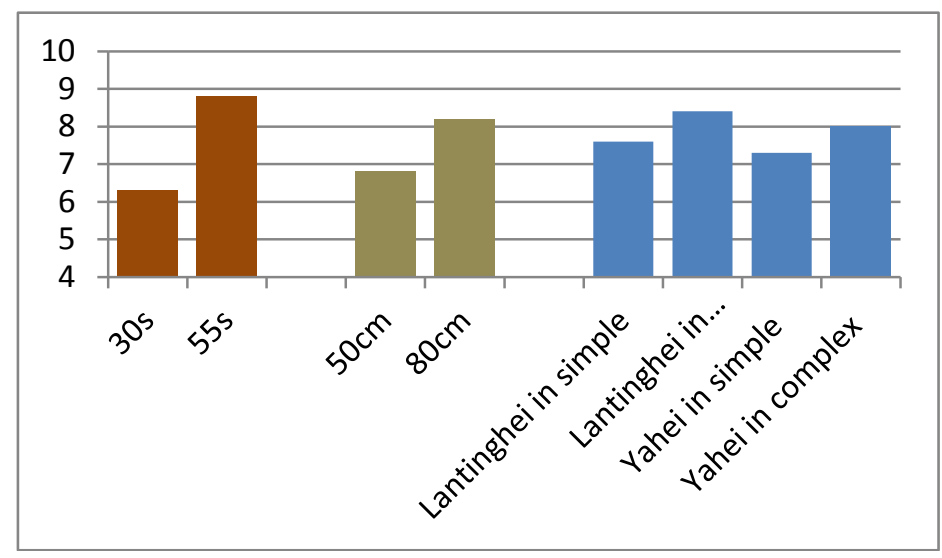

Fig. 2. The results of the main effects of 'minimum character size for $100 \%$ correctness'

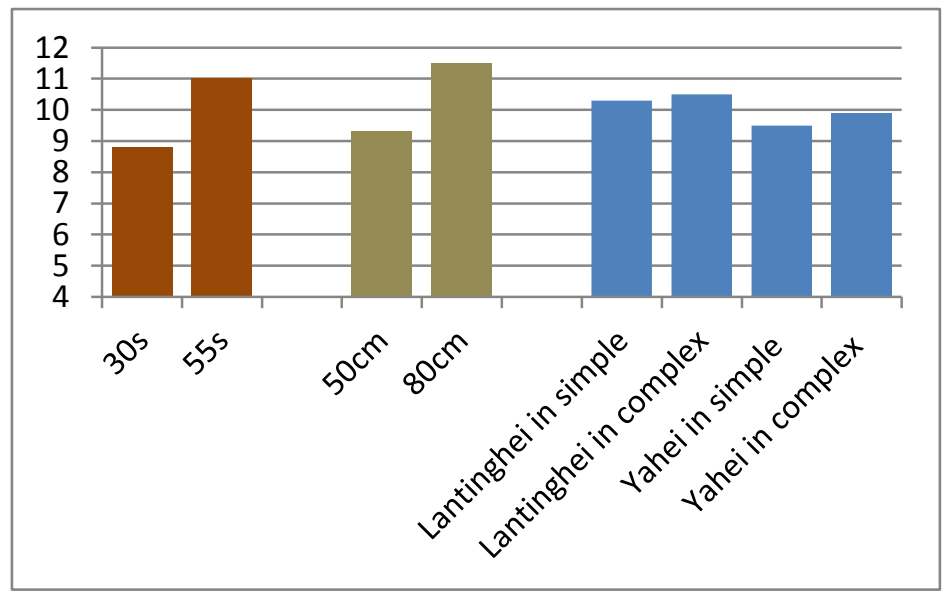

Fig. 3. The results of the main effects of' minimum character size for the least comfort'

\section{Discussion}

The present study investigated the effects of age, viewing distance and font type on two dependent variables: (1) 'minimum character size for $100 \%$ correctness'; (2) 'minimum character size for the least comfort'. 
With regard to age, the young groups showed better legibility than elderly groups. The average character sizes for the two dependent variables of the group of subjects in their 55s were larger than those of the group of subjects in their 30s. That is, the average character sizes for subjects in their $55 \mathrm{~s}$ were $6.3 \mathrm{pt}$ and $8.8 \mathrm{pt}$ for the 'minimum character size for $100 \%$ correctness' and 'minimum character size for the least comfort', respectively, whereas those of subjects in their 30 s were $8.8 \mathrm{pt}$ and $11.0 \mathrm{pt}$, respectively. These findings might be explained by a reduction of light on the retina as well as the increasing of age.

According to the comparison analyses for correctness and comfort, the 'minimum character sizes for the least comfort' were larger than the 'minimum character sizes for $100 \%$ correctness'. This means that the character sizes that caused the least discomfort were significantly different from the character sizes at which people read with $100 \%$ correctness. Generally people preferred larger characters than the sizes at which people can nevertheless read with perfect accuracy. Therefore, performance as well as subjective preference should be considered when recommending character sizes for Chinese words to improve legibility, because even if the recommended character sizes ensure that they can be read correctly, people might still feel discomfort when reading.

\section{Conclusions}

This study examined the effects of age, viewing distance and font type on two dependent variables to evaluate the legibility of Chinese characters. A summary of the main findings is as follows:

Age: The young group (6.3 pt) could read character sizes smaller than those of the elderly group ( $8.8 \mathrm{pt}$ ) for the 'minimum character size for $100 \%$ correctness', respectively. In addition, the young group $(8.8 \mathrm{pt})$ could read significantly smaller character sizes than those of the elderly group $(11.0 \mathrm{pt})$ for the 'minimum character size for the least comfort', respectively.

Viewing distance: $50 \mathrm{~cm}(6.8 \mathrm{pt})$ allowed for character sizes about2pt smaller than those at $80 \mathrm{~cm}(8.2 \mathrm{pt})$ for the 'minimum character size for $100 \%$ correctness', respectively. In addition, $50 \mathrm{~cm}(9.3 \mathrm{pt})$ allowed for character sizes about $2 \mathrm{pt}$ smaller than those required at $80 \mathrm{~cm}(11.5 \mathrm{pt})$ for the 'minimum character size for the least comfort', respectively.

This study might be helpful in that they provide basic information with which to determine the legibility of Chinese characters.

\section{References}

1. Wang, J., Shao, Z.F.: Size Threshold of Chinese Characters and Appropriate Character space in Electronic Map. Chinese Journal of Applied Psychology 114(1), 060-065 (2008)

2. Zhou, A., Zhang, X., Shu, H., He, L.G.: Cognitive Processes Based on the Font, Size and Characteristics in Chinese Two-Character. Psychological Science 32(1), 134-136 (2009)

3. Guidelines for the elderly and people with disabilities - visual signs and displays - estimation of minimum legible size for a Japanese single character. Japanese Industrial Standard, JIS S 0032:2003 (2003) 
4. Guidance for industry: Labeling OTC human drug products questions and answers. Washington, DC: Food and Drug Administration. US Department of Health and Human Services (2005)

5. Wang, Y., Shao, Z.: Stroke Frequency and Font Effects on Chinese Character Recognition Threshold. Psychological Science 32(1), 134-136 (2009)

6. Kong, Y.-K., Lee, I., Jung, M.-C., Song, Y.-W.: The effects of age, viewing distance, display type, font type, colour contrast and number of syllables on the legibility of Korean characters. Ergonomics 54(5), 453-465 (2011)

7. Jin, W., Zhu, Z., Shen, M.: The effect of fonts of characters on recognition. Chinese Journal of Applied Psychology 7(2) (1992)

8. Zhang, J.Y., Zhang, T., Xue, F., Liu, L., Yu, C.: Legibility variations of Chinese characters and implications for visual acuity measurement in Chinese reading population. Investigative Ophthalmology \& Visual Science 48(5), 2383-2390 (2007) 